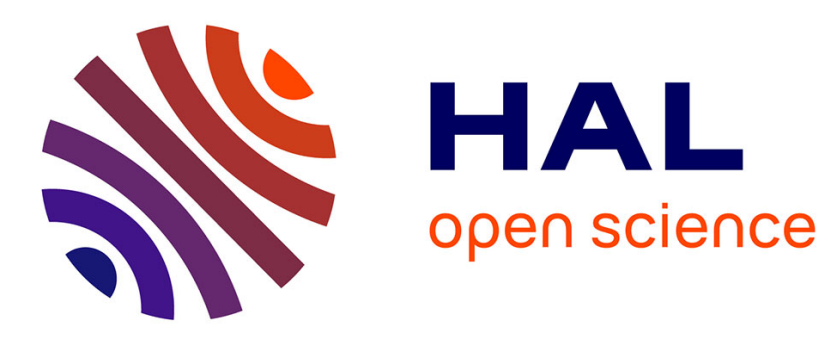

\title{
Title: Late cortical disinhibition in relaxed versus active hand muscles
}

Alexandre Caux-Dedeystère, Philippe Derambure, H. Devanne

\section{To cite this version:}

Alexandre Caux-Dedeystère, Philippe Derambure, H. Devanne. Title: Late cortical disinhibition in relaxed versus active hand muscles. Neuroscience, 2015, 298, pp.52-62. 10.1016/j.neuroscience.2015.04.018 . hal-03185935

\section{HAL Id: hal-03185935 \\ https://hal.science/hal-03185935}

Submitted on 30 Mar 2021

HAL is a multi-disciplinary open access archive for the deposit and dissemination of scientific research documents, whether they are published or not. The documents may come from teaching and research institutions in France or abroad, or from public or private research centers.
L'archive ouverte pluridisciplinaire HAL, est destinée au dépôt et à la diffusion de documents scientifiques de niveau recherche, publiés ou non, émanant des établissements d'enseignement et de recherche français ou étrangers, des laboratoires publics ou privés. 
Title: Late cortical disinhibition in relaxed versus active hand muscles.

Authors: Alexandre Caux-Dedeystère ${ }^{1}$, Philippe Derambure ${ }^{1}$ and Hervé Devanne ${ }^{1,2}$

1. Université de Lille, EA 1046, Centre Hospitalier Régional Universitaire de Lille, Neurophysiologie Clinique, F-59037 Lille cedex, France

2. Université du Littoral Côte d'Opale, F-62228 Calais cedex, France

24 pages, 6 figures, 2 tables

Corresponding author: Hervé Devanne

CHRU de Lille

Neurophysiologie Clinique

Hôpital Roger Salengro

F-59037 Lille cedex, France

Tél : 33320446354

Fax : 33320446355

Email : herve.devanne@chru-lille.fr 


\section{Abstract}

Recent research suggests that long-interval intracortical inhibition (LICI) is followed by a transitory period of late cortical disinhibition (LCD) that can even lead to a net increase in cortical excitability. The relationship between LICI/LCD and voluntary drive remains poorly understood. Our study aims at investigating the influence of index abduction on LICI and LCD in an actively engaged muscle and a neighboring muscle, while varying the intensity of the conditioning stimulus. Motor-evoked potentials (MEPs) were recorded from the first dorsal interosseus (FDI) and abductor digiti minimi (ADM) muscles in 13 subjects. Paired-pulses were delivered with 10 different interstimulus intervals (ranging from $60 \mathrm{~ms}$ to $290 \mathrm{~ms}$ ). Whatever the condition (relaxed or active FDI), the test stimulus was set to evoke an MEP of $1 \mathrm{mV}$. The time course of conditioned MEPs amplitude was compared for relaxed and active conditions when the conditioning stimulus (CS) intensity was set to (i) $130 \%$ of the rest motor threshold (RMT) or (ii) to evoke the same size of MEP under both conditions. LICI lasted longer (i.e. disinhibition occurred later) at rest than during abduction when evoked either by similar or matched conditioning stimuli. No post-LICI facilitation was observed at rest - even when the CS intensity was set to $160 \%$ RMT. In contrast, long-interval intracortical facilitation was observed in the quiescent ADM when the FDI was active. Long-interval intracortical facilitation may then be associated with voluntary activity albeit with a lack of topographic specificity.

Keywords: motor cortex, late cortical disinhibition, long-interval intracortical facilitation, voluntary activity 
Abbreviations: EMG, electromyogram; MEP, motor-evoked potential; FDI, first dorsal interosseus; GABA, gamma aminobutyric acid; ISI, interstimulus interval, LCD, late cortical disinhibition; LICF, long-interval intracortical facilitation; LICI, long-interval intracortical inhibition; SICI, short-interval intracortical inhibition; MVC, maximal voluntary contraction; TMS, transcranial magnetic stimulation; CS, conditioning stimulus; ADM, abductor digiti minimi; RMT, resting motor threshold.

\section{Acknowledgements}

We thank Jean-Louis Bourriez and François Cassim for helpful comments and David Fraser for editorial support. 


\section{Introduction}

Over the past two decades, many studies have used paired-pulse transcranial magnetic stimulation (TMS) protocols to study intracortical inhibitory and facilitatory networks and the latter's underlying relationship with motor behavior. It has been suggested that intracortical inhibitory and excitatory mechanisms have a role in the modulation of cortical excitability prior to and during voluntary movements, so that corticospinal drive meets the task's requirements (Floeter and Rothwell, 1999). For example, the shortinterval intracortical inhibition (SICI) that can be observed when a subthreshold conditioning stimulus is delivered 1 to $4 \mathrm{~ms}$ before the test pulse has been extensively investigated. After SICI was first evidenced at rest (Kujirai et al., 1993), it was found that this intracortical inhibition decreases before movement onset (Reynolds and Ashby, 1999) and during tonic voluntary contraction (Ridding et al., 1995; Ortu et al., 2008). Furthermore, SICI is weaker during complex tasks (Kouchtir-Devanne et al., 2012) than during simple isometric contraction and thus is thought to correspond to a mechanism by which cortical motor neuron activity is fine-tuned to allow precise movements. The mechanism referred to as long-interval intracortical inhibition (LICI) was observed initially by Valls-Solé et al. (1992) and later by Wassermann et al (Wassermann et al., 1996) with interstimulus intervals (ISIs) ranging from 50 and $200 \mathrm{~ms}$. Although LICI was not as extensively investigated as SICI, several studies have highlighted its potential significance in motor control. For example, LICI evoked in the first dorsal interosseus (FDI) muscle is weaker when the muscle is engaged in a thumb-index precision grip than during index abduction (Kouchtir-Devanne et al., 2012). LICI also appears to be shorter and weaker during active contraction than at rest (Wassermann et al., 1996; Chen et al., 1997). More recent studies (using paired TMS protocols) have also revealed that LICI is progressively suppressed at longer ISIs and may even be followed by a 
period of long-interval intracortical facilitation (LICF), corresponding to a net increase of cortical excitability. Late cortical disinhibition (LCD) is a newly reported phenomenon that is thought to reflect the period of time during which long-interval intracortical inhibition (which decreases more or less rapidly) is combined with the possible recruitment of facilitatory cortical interneurons responsible for the subsequent LICF. The LCD has been reported to occur at rest (Cash et al., 2010, 2011) and during voluntary activity (Caux-Dedeystère et al., 2014), and may reflect post-movement synchronization of facilitatory cortical interneurons (Cash et al., 2010). Recent evidence suggests that the induction of long-term potentiation plasticity is facilitated when repetitive TMS pulses are delivered during the disinhibition period (Cash et al., 2014). Better knowledge of the LCD's time-dependent profile is therefore of particular value for further improving the efficacy of repetitive TMS protocols.

The time profiles of LICI/LCD at rest and during active contraction have not previously been compared. The primary objective of the present study was thus to analyze the influence of index tonic voluntary abduction on LICI and LCD in the FDI at different conditioning stimulus intensities. We also investigated the topographic specificity of LCD and LICF by recording the responses of a neighboring muscle (the abductor digiti minimi, ADM) not engaged in the index abduction task. Throughout the text, the conditioned MEP's return to baseline i.e. the weakening of LICI will be referred to as LCD. Post-LCD facilitation (corresponding to the net increase in cortical excitability after LCD, when present) will be referred to as LICF (see figure 1). 


\section{Methods}

\section{Participants}

Thirteen subjects (including six females) aged between 20 and 46 (mean \pm SD age: 24.92 \pm 7.26 ) participated in the study after providing their written, informed consent. None of the subjects was taking any medication or was suffering from a neurological or psychiatric disorder. The study was approved by the local independent ethics committee (Comité Consultatif de Protection des Personnes Nord Ouest, Amiens, France) and complied with the tenets of the Declaration of Helsinki.

\section{Electromyographic recordings}

Participants were seated in a comfortable chair, with their forearms resting on a heightadjustable table. $\mathrm{Ag}-\mathrm{AgCl}$ surface electrodes were used to record the electromyographic (EMG) activity of the FDI and ADM muscles of the dominant hand in a belly-tendon montage. The electrodes were positioned over the body of each the two muscles and (for the ADM and FDI, respectively) the first metacarpophalangeal joint of the fifth finger or the thumb. A large ground electrode was attached to the wrist. The EMG signals were amplified (x1000), high-pass filtered at $10 \mathrm{~Hz}$ and low-pass filtered at $1000 \mathrm{~Hz}$ (Digitimer, Hertfordshire, UK) prior to sampling at $2 \mathrm{kHz}$ with a 1401 MicroMKII device (Cambridge Electronic Design, Cambridge, UK). The data were stored on a computer for subsequent off-line analysis using customized SIGNAL software (Cambridge Electronic Design). The subjects first produced maximal voluntary contraction (MVC) of the FDI (abduction of the index finger) in three successive trials. A 1401+ device and customized SPIKE2 software (both from Cambridge Electronic Design) were used to record and display the root mean square of the low-pass filtered $(100 \mathrm{~Hz})$ EMG signal from the FDI as a percentage of the MVC EMG signal. 


\section{Stimulation procedures}

TMS was performed using a figure-of-eight focal coil (external diameter: $9.5 \mathrm{~cm}$ ) connected to two Magstim 200 stimulators (The Magstim Company Ltd, Whitland, UK) via a Bistim module. Stimulation was applied over the optimal scalp point for the FDI of the dominant hand, i.e. the site that yield the strongest MEPs for the FDI at a given suprathreshold intensity. This scalp point was determined by moving the coil while the subject relaxed his/her hand muscles. To ensure constant coil positioning throughout the series, the FDI's hotspot was marked on a swimming cap worn by the subject. The coil was held tangentially to the scalp, with the handle pointing backwards and laterally (at a $45^{\circ}$ angle from the midline). We then measured the resting motor threshold (RMT) of the FDI, defined as the lowest possible stimulus intensity capable of eliciting MEPs $>50 \mu \mathrm{V}$ in at least 5 of 10 trials in the muscle at rest. In all experimental series, single pulses and dual pulses were separated by an inter-trial interval of random duration (between 4 and $6 \mathrm{~s}$ ).

\section{Experimental design}

In the first two series (one at rest and one with index abduction, in randomized order), the intensity of the conditioning stimulus $(\mathrm{CS})$ was set to $130 \% \mathrm{RMT}\left(\mathrm{CS}_{130}\right)$. The mean amplitude of the motor response evoked by $\mathrm{CS}_{130}$ was measured after each of the two series. Given that motor thresholds are lower during active motor tasks, a fixed conditioning stimulus $\left(\mathrm{CS}_{130}\right)$ is likely to recruit more neurons during the active task (as suggested by greater MEPs) than at rest. We thus sought to determine, if exist, whether the differences between active and relaxed muscles were due (at least in part) to a differential effect of the conditioning stimulus on the neural circuits. To this end, the 
participants performed two additional series. In the first series, the intensity of the CS in the abduction task was lowered ( $\mathrm{CS}^{*}{ }_{\text {Lower }}$ ), so that it evoked an MEP whose amplitude matched that induced by $\mathrm{CS}_{130}$ at rest. In the second series, we increased the CS intensity in the rest condition (CS* ${ }_{\text {Higher }}$ ) so that it induced an MEP whose amplitude matched that induced by $\mathrm{CS}_{130}$ in the abduction task (Figure 2).

Whatever the series, the test stimulus intensity was set to induce an MEP of about $1 \mathrm{mV}$ (measured peak-to-peak) when delivered alone. On the basis of our previous results (Caux-Dedeystere et al, 2014), ISIs were chosen in order to study post-LICI disinhibition with accuracy. Ten ISIs $(60,90,120,140,150,165,180,195,230$ and $290 \mathrm{~ms})$ were applied in random order in two sets of five. This was performed in order to shorten the duration of the block and avoid muscle fatigue during the tasks. In summary, a total of eight series (two series of 5 ISIs $\mathrm{x}$ two conditioning intensities $\mathrm{x}$ two conditions) were performed. In each of the series, eight conditioned responses were recorded in pseudorandom order for each ISI, together with eight non-conditioned responses delivered pseudo-randomly throughout the block. Each series lasted for about three minutes, and none of the subjects reported fatigue or difficulties in maintaining the level of muscle contraction (10\% of MVC) during index abduction. In all series, the subject was instructed to fully relax the ADM muscle. The ADM's activity was monitored throughout the experiment; when necessary, the subject was reminded that the ADM should not be contracted.

\section{Data analysis}

The mean conditioned peak-to-peak MEP amplitude was measured offline and expressed as a percentage of the non-conditioned MEP. Statistical analysis was carried out with SPSS v16.0 software (IBM Corp., Armonk, NY). The effects of ISI and task were 
evaluated in a two-way repeated-measures analysis of variance (RM-ANOVA), with two factors: task (rest, abduction) and ISI (10 levels). Three comparisons were made: (i) abduction with $\mathrm{CS}_{130}$ vs. rest with $\mathrm{CS}_{130}$; (ii) abduction with $\mathrm{CS}_{130}$ vs. rest with $\mathrm{CS}^{*}{ }_{\text {Higher }}$ and (iii) abduction with $\mathrm{CS}^{*}$ Lower vs. rest with $\mathrm{CS}_{130}$. If the RM-ANOVA revealed a significant effect of one or both factors and/or a significant interaction between the two factors, a post hoc test with Bonferroni correction was performed to determine the ISIs for which the two conditions differed. If the effect of ISI was significant, the difference from baseline (i.e. the non-conditioned MEP) was tested for each ISI in a one-sample ttest. Unconditioned MEPs evoked either by $\mathrm{CS}_{130}, \mathrm{CS}^{*}{ }_{\text {Lower, }} \mathrm{CS}^{*}{ }_{\text {Higher }}$ or Test stimulus were compared with a paired t-test (or a Wilcoxon signed-rank test depending on the distribution and variance of the data distribution). The same test was used to study the influence of CS intensity on conditioned MEPs at given ISIs. In all analyses, the threshold for statistical significance was set to $\mathrm{p}<0.05$. 


\section{Results}

1. Comparison of LICI/LCD profiles in the resting condition versus index abduction, with $\mathrm{CS}_{130}$

The mean RMT across all subjects was $40.61 \pm 4.56 \%$. The mean MEP amplitude elicited in the FDI by $\mathrm{CS}_{130}$ was $2.08 \pm 1.34 \mathrm{mV}$ at rest and $5.53 \pm 1.73 \mathrm{mV}$ in the abduction task (Figure 2). The mean TS MEP amplitude was $1.12 \pm 0.33 \mathrm{mV}$ at rest and $1.15 \pm 0.32 \mathrm{mV}$ during abduction.

Figure 3A shows the time course of LICI and LCD in the FDI at rest and during abduction, with $\mathrm{CS}_{130}$. The RM-ANOVA revealed (i) significant effects of both task $(\mathrm{F}=24.36$, $\mathrm{p}<0.01)$ and ISI $(\mathrm{F}=17.13, \mathrm{p}<0.01)$ and (ii) a significant interaction between the two ( $\mathrm{F}$ $=5.80, \mathrm{p}<0.01)$. Two main intercondition differences are illustrated in figure 3A. Firstly (and in line with Wasserman et al.'s findings (Wassermann et al., 1996)), we found that LICI decreased more rapidly in the abduction task than at rest. In the former condition, LICI began to decrease from $90 \mathrm{~ms}$ and the conditioned MEP crossed the baseline around 165 ms. At rest, inhibition was still significantly present and lasted until $180 \mathrm{~ms}$. Secondly, the amplitude of the conditioned MEPs was significantly greater during abduction than at rest at the longest ISIs (from $165 \mathrm{~ms}$ to $290 \mathrm{~ms}$ ). Although disinhibition was followed by marked facilitation when the FDI was activated, the conditioned MEP never exceeded the baseline level when the muscle was at rest (table 1). In the latter condition, LICI was followed by clear disinhibition from 195 ms to 230 ms. However the conditioned MEP was significantly lower than baseline $(p<0.01)$ at the longest ISI (290 ms). 
The nature of the relationship between LICI/LCD and ISI was almost the same for the quiescent ADM (Figure 3B) as it was for the FDI. At the shortest ISIs, LICI was observable (regardless of the intensity of FDI contraction) and disinhibition of the ADM also occurred earlier when the FDI muscle was tonically activated (although the ADM remained quiescent under both conditions). In line with the data for the FDI, disinhibition in the ADM was not followed by a phase of MEP facilitation when the muscles were at rest. The most striking observation was that although the ADM muscle was always kept at rest, disinhibition was followed by strong facilitation when the FDI muscle was tonically activated during index abduction (Figure 3B, Table2). When comparing FDI activation and rest, the ADM's conditioned MEPs differed significantly at all ISIs between $165 \mathrm{~ms}$ and $230 \mathrm{~ms}$.

\section{Comparison of LICI/LCD profiles with $C S_{130}$ in the resting condition and adjusted CS intensity (CS* $\left.{ }^{*}{ }_{\text {Lwer }}\right)$ in the abduction task.}

For the study participants as a whole, the mean \pm SE CS* Lower intensity was $102.17 \pm 3.21 \%$ RMT (41.62 $\pm 2.01 \%$ of the stimulator output). The mean MEP amplitude evoked by $\mathrm{CS}^{*}$ Lower in the abduction task was $2.15 \pm 1.22 \mathrm{mV}$ and did not differ significantly from that obtained at rest with $\mathrm{CS}_{130}(2.08 \pm 1.34 \mathrm{mV})$ (Figure 2). The TS MEP amplitude was respectively $1.12 \pm 0.33 \mathrm{mV}$ and $1.10 \pm 0.21 \mathrm{mV}$ in resting and abduction conditions. Reducing the CS intensity did not appear to greatly modify the curve obtained in the abduction task (Figure 4A). The curves obtained with matched CS under the two conditions were clearly distinct following the initial LICI phase observed at the lowest ISIs. This was confirmed by the RM ANOVA, which revealed (i) significant effects of both task $(\mathrm{F}=24.36, \mathrm{p}<0.01)$ and ISI $(\mathrm{F}=17.13, \mathrm{p}<0.01)$ and (ii) a significant interaction 
between the two $(F=5.80, p<0.01)$. Although the use of a lower CS intensity resulted in less facilitation than with $\mathrm{CS}_{130}$ during abduction, the facilitation remained statistically significant (relative to baseline intensity) at $180 \mathrm{~ms}$ and $195 \mathrm{~ms}$ (table 1). When compared with the resting condition, disinhibition in abduction again occurred earlier, and LICI had disappeared by $140 \mathrm{~ms}$ with $\mathrm{CS}^{*}$ Lower. The two conditions differed significantly in terms of the conditioned MEPs at all ISIs between 120 ms and $290 \mathrm{~ms}$.

The time-dependent profile of the conditioned MEP obtained from the ADM was very similar to that of the FDI when $\mathrm{CS}^{*}$ Lower was used (Figure 4B). Compared with the rest condition, disinhibition also occurred earlier in the ADM and was followed by a phase of facilitation being significant at ISI $180 \mathrm{~ms}$ (Table 2).

3. Comparison of LICI/LCD profiles with $\mathrm{CS}_{130}$ in the abduction task and an adjusted CS intensity (CS* Higher) in the resting condition.

In 5 of the 13 subjects, a ceiling effect prevented us from adjusting the FDI MEP amplitude evoked by $C S^{*}$ Higher to that obtained during abduction with $\mathrm{CS}_{130}$. In the remaining 8 subjects, the mean FDI MEP amplitude evoked by $C S^{*}{ }_{\text {Higher }}$ was $4.34 \pm 1.85$ $\mathrm{mV}$ and did not differ significantly from that obtained during abduction with $\mathrm{CS}_{130}$ $\left(4.58 \pm 1.46 \mathrm{mV}\right.$ ) (Figure 2). For the 8 participants, the mean $C S^{*}{ }_{H i g h e r}$ intensity was $161.79 \pm 9.01 \%$ RMT $(65.88 \pm 2.47 \%$ of the stimulator output). The TS MEP amplitude was respectively $1.16 \pm 0.26 \mathrm{mV}$ and $1.25 \pm 0.29 \mathrm{mV}$ in resting and abduction conditions. Figure 5A shows the time course of LICI and LCD under each of the two conditions; the abduction condition was the same as that in figure 3A but the curve was drawn with data from 8 of the 13 subjects). Adjusting the CS intensity in the rest condition did not 
lead to significant changes in the time-dependent profile of conditioned FDI MEPs. As in the comparisons described above, the RM ANOVA indicated (i) significant effects of both task $(\mathrm{F}=20.58, \mathrm{p}<0.01)$ and ISI $(\mathrm{F}=11.87, \mathrm{p}<0.01)$ and (ii) a significant interaction between the factors $(\mathrm{F}=4.60, \mathrm{p}<0.01)$. While the use of CS intensity as high as 1.6 times the RMT delayed disinhibition, we did not notice any facilitation at the longest ISIs in the rest condition; this contrasted with the strong facilitation observed during abduction (Figure 5A, table 1).

In the neighboring ADM muscle, the application of $C S^{*}$ Higher was associated with longer LICI and greater disinhibition (Figure 5B). Again, there was no facilitation of the ADM's conditioned MEPs when both muscles were at rest.

\section{Influence of CS intensity}

In the quiescent FDI, increasing the CS intensity from 130\% RMT to $160 \%$ RMT only induced subtle changes: (i) the LICI was deeper, but the difference was only significant at ISI $165 \mathrm{~ms}$, and (ii) the LCD tended to occur later (Figure 6A). Although conditioned MEPs tended to be greater with CS* ${ }_{\text {Higher }}$ at longest ISIs (i.e. $230 \mathrm{~ms}$ and $290 \mathrm{~ms}$ ) there was no difference due to the CS intensity. In contrast, marked changes were observable in the active FDI muscle when the CS intensity was decreased from $130 \%$ RMT to just above threshold ( 102\% RMT)(Figure 6B). At first, the LICI was significantly lower, and the LCD occurred earlier. Furthermore, although LICF was still present with CS* ${ }_{\text {Lower, }}$ it lasted shorter and the amount of LICF was weaker than with $\mathrm{CS}_{130}$. The influence of CS intensity on the ADM muscle was quite similar. Increasing the CS intensity did not induce significant change in LICI and LCD when the muscles were at rest. The reduction 
of CS intensity when the FDI was active was accompanied by a decrease in the amount of LICI between $60 \mathrm{~ms}$ and $120 \mathrm{~ms}$ in the ADM muscle. 


\section{Discussion}

The purpose of this study was to compare the time profiles of LICI/LCD at rest and during active contraction. We thus analysed the influence of index abduction on LICI and LCD in the FDI and in a neighboring muscle, the ADM, at different conditioning intensities. We have shown that (i) the duration of LICI was longer at rest than during contraction; (ii) LCD was observed at rest and during tonic voluntary contraction, whereas LICF only occurred during the active task; (iii) the overall time-dependent evolution from LICI to LCD/LICF was not influenced by CS intensity; (iv) but the "size" of LICI, LCD, or LICF may be influenced by CS intensity; and finally (v) similar results were observed in a muscle adjacent to the contracted muscle, but not formally involved in the contraction task. In the discussion that follows, we begin by addressing the changes of duration and amount of LICI that accompany voluntary FDI activation. We then consider the neural mechanisms that may underlie the changes in LCD and LICF occurring when a muscle becomes active and when the conditioning intensity increases. Lastly, we consider the late phase of inhibition that was observable in the quiescent FDI muscle at longest interstimulus intervals.

\section{$\underline{\text { LICI in active versus relaxed muscle }}$}

To the best of our knowledge, the time course of LICI (both at rest and during voluntary contraction) has only been reported on twice (Wassermann et al., 1996; Chen et al., 1997) but has never been explored in the post-LICI period during which LCD and LICF are observable. Our data are consistent with these previous observations in that (regardless of the amount of inhibition) the LICI lasted longer when the muscle was relaxed than during tonic, voluntary condition. The data recorded during FDI voluntary 
contraction show more obviously than at rest that increasing the conditioning intensity is accompanied by more ample, earlier disinhibition. Although LICI appeared to be most intense when the CS intensity increased (from $130 \%$ to $160 \%$ RMT in the relaxed FDI and from $102 \%$ to $130 \%$ RMT during index abduction), the degree of inhibition was much the same, at least until ISI $90 \mathrm{~ms}$ in the active and quiescent FDI. This finding suggests that unlike SICI which falls during voluntary muscle contraction (Ridding et al., 1995; Ortu et al., 2008) LICI does not participate in the down-regulation involved in the recruitment of new cortical units. At first sight, these results appear to contrast with certain literature data showing that LICI is lower during voluntary activity than at rest and decreases even further as the level of muscle contraction increases (Hammond and Vallence, 2007). In the latter study, however, LICI was only measured at an ISI of 100 ms, and it is likely that disinhibition already occurs at this interval - as suggested by the report that LICI can turn into facilitation when subthreshold conditioning stimuli are applied (Vallence et al., 2014).

\section{$\underline{\text { LCD in active versus relaxed muscle }}$}

To date, very few studies have explored excitatory and inhibitory phenomena in the post-LICI period. Regardless of the amplitude of the preceding LICI, the disinhibition process began earlier in the active task. The subsequent, conditioned MEPs returned to the baseline level more rapidly and were even clearly facilitated during index abduction. The mechanisms responsible for LCD (i.e. a decrease in LICI and the conditioned MEP's return to baseline) have not yet been fully characterized. One of the most convincing hypothesis proposed to date holds that the conditioning pulse induces (i) immediate, inhibitory effects (SICI and LICI) mediated by postsynaptic gamma aminobutyric acid (GABA) receptors and (ii) delayed disinhibition of SICI neurons mediated by activation 
of presynaptic GABA-B receptors, which may be responsible (at least in part) for the LCD (Cash et al., 2010). Interaction between LICI and SICI circuits (Sanger et al., 2001) may constitute the physiological substrate for this type of disinhibition. However, this hypothesis holds that disinhibition would not occur (or would occur to a lesser extent) when SICI neurons have been depressed as during voluntary activation (Ridding et al., 1995; Ortu et al., 2008). In such a case, and given that SICI neurons are less active, the removal of their inhibition should be accompanied by weaker disinhibition. In contrast, our data show earlier disinhibition with more rapid decay during the active task compared with rest. We thus consider that another mechanism is likely to be involved in the rapid decrease in LICI (and the increase in LICF) during voluntary contraction. One possible candidate is contraction-induced afferent input. During index abduction, many proprioceptive endings are stimulated and may then modulate corticospinal excitability. A large body of literature data suggests that information from skin and muscle afferents can interact with inhibitory networks in the primary motor cortex and thus modify motor output (Hess et al., 1999; Ridding and Rothwell, 1999; Rosenkranz and Rothwell, 2004). Although speculative, our hypothesis is supported by the finding that median nerve stimulation may decrease the intensity of LICI (Sailer et al, 2002; Udupa et al, 2009) and that in half of the subjects with LICI and long-latency afferent inhibition, LICI turned into facilitation when paired with peripheral median nerve stimulation (Sailer et al, 2002).

\section{LICF in active versus relaxed muscle}

Our most surprising finding (and in contrast to the report by Cash et al., 2010, 2011) was the absence of LICF in all subjects when both muscles were at rest. This result was especially unexpected because we used much the same stimulation parameters as Cash 
et al. (2010), namely a conditioning intensity set to $130 \%$ RMT, a test intensity set to evoke an MEP of about $1 \mathrm{mV}$, the same range of ISIs, and a similar muscle. Furthermore, increasing the CS intensity to $160 \%$ RMT in the resting condition was not effective in inducing LICF in our experiments, whereas increasing the conditioning stimulus intensity from $110 \%$ to $140 \%$ in Cash et al.'s study delayed the inhibition-to-facilitation inflection point and increased the net cortical excitability above baseline (Cash et al., 2010). The reasons for these discrepancies remain unclear. Even though differences between subject populations are unlikely, they cannot be ruled out. However, given the clear facilitation observed in the quiescent ADM muscle when the FDI became active (see Figures 3B \& 4B), an alternative hypothesis could be that the recruitment of a muscle is accompanied by diffuse effects in neighboring muscles -leading to the LICF observed in both hand muscles even when only one muscle was active. If this hypothesis is confirmed by additional experiments, it would mean that LICF might arise in a resting muscle as soon as other neighboring muscles are activated even slightly. Lastly, it is noteworthy that only 8 subjects were involved in this part of the study; hence, the power of the statistical test was reduced accordingly. However, when looking at individual datasets, only 4 of the 8 subjects having received the highest CS intensity showed facilitation of the conditioned MEPs at an ISI of $230 \mathrm{~ms}$. The remaining 4 subjects had less intense conditioned than unconditioned MEPs. At the previous ISI (195 ms) and following ISI (290 ms), the conditioned MEP was facilitated in only 1 of the 8 subjects. Even though LICF might conceivably be present at rest in some subjects at an ISI around $230 \mathrm{~ms}$, it is not likely to be intense.

\section{Influence of CS intensity}


In line with some previous studies, our data seems at first sight to confirm that LICI is affected by CS intensity. The amount of LICI was de facto greater when the CS intensity increased in both relaxed and active FDI muscle and LICI tended to last longer with increasing CS, at least in the active condition. This is in line with Vallence et al's finding that the CS threshold for evoking LICI with an interstimulus interval of $100 \mathrm{~ms}$ is lower than that for evoking LICI with an ISI of 150ms (Vallence et al, 2014). The corollary of LICI prolongation with increasing CS intensity was that LCD occurred later, as already reported in a previous study showing that the inflection point (i.e. the change of slope during the transition between inhibition and facilitation) was delayed (Cash et al, 2010), or, in the present work, by the rightward shift of the curve with more intense CS intensity. In the active condition but not at rest, we found that the amount of facilitation following LCD was dependent on CS intensity with LICF being observable with CS just above the resting motor threshold. Taken together, these data suggest that the conditioning stimulus exerts some complex effects on both inhibitory circuits responsible for LICI, LCD and most likely on excitatory neurons that could be recruited preferentially during voluntary contraction.

\section{Delayed inhibition in relaxed muscles}

When both the ADM and FDI were at rest, we observed a late inhibition phase in the FDI muscle (as evidenced by the significant inhibition of the conditioned MEPs at the longest ISI, i.e. $290 \mathrm{~ms}$ ). A quite similar profile was observed for the ADM curve; after disinhibition occurred, the conditioned MEP slightly decreased at an ISI of $290 \mathrm{~ms}$. To the best of our knowledge, conditioned MEPs have never been measured at such a long ISI. The late inhibition observed here is unlikely to be an artifact, since it was present in all the participants and varied little from one participant to another. Although the nature 
of this late inhibition remains to be assessed, we hypothesize that LICI lasts longer ( $>290 \mathrm{~ms}$ ) than the generally assumed limit of around $200 \mathrm{~ms}$ and is attenuated in the time window corresponding to disinhibition (i.e. between $150 \mathrm{~ms}$ and $300 \mathrm{~ms}$ ). Very slow phenomena of this type have been evidenced in rat and human cortical neurons (Deisz, 1999a). They are mediated by GABAB receptors, the activity of which increases the potassium conductance underlying slow, inhibitory postsynaptic potentials lasting up to $400 \mathrm{~ms}$ (Deisz, 1999b). Our results are thus consistent with slow, long-lasting LICI that is mediated by a post-synaptic $\mathrm{GABA}_{\mathrm{B}}$ effect and attenuated by a disinhibition phase due to presynaptic inhibition of GABA $\mathrm{A}_{\mathrm{A}}$ terminals. Nevertheless, as evoked earlier, the net increase in cortical excitability (the LICF) observed during index abduction is unlikely to be only due to SICI disinhibition.

In conclusion, our study is the first to show that rest and voluntary hand muscle activity differ in terms of the time course of LICI, LCD and LICF. Given the lack of LICF and the slower disinhibition observed at rest, we speculate that an intracortical excitatory component (tonically inhibited at rest but released when the muscle becomes active) contributes to the reduction in the late part of LICI and to the strong increase in net cortical excitability. Although speculative, the involvement of sensorial inputs in LCD and LICF merits to be investigated. 


\section{Bibliography}

Cash RFH, Murakami T, Chen R, Thickbroom GW, Ziemann U (2014) Augmenting Plasticity Induction in Human Motor Cortex by Disinhibition Stimulation. Cereb Cortex N Y N 1991.

Cash RFH, Ziemann U, Murray K, Thickbroom GW (2010) Late cortical disinhibition in human motor cortex: a triple-pulse transcranial magnetic stimulation study. J Neurophysiol 103:511-518.

Cash RFH, Ziemann U, Thickbroom GW (2011) Inhibitory and disinhibitory effects on Iwave facilitation in motor cortex. J Neurophysiol 105:100-106.

Caux-Dedeystère A, Rambour M, Duhamel A, Cassim F, Derambure P, Devanne H (2014) Task-dependent changes in late inhibitory and disinhibitory actions within the primary motor cortex in humans. Eur J Neurosci 39:1485-1490.

Chen R, Wassermann EM, Caños M, Hallett M (1997) Impaired inhibition in writer's cramp during voluntary muscle activation. Neurology 49:1054-1059.

Deisz RA (1999a) GABAB receptor-mediated effects in human and rat neocortical neurones in vitro. Neuropharmacology 38:1755-1766.

Deisz RA (1999b) The GABA(B) receptor antagonist CGP 55845A reduces presynaptic GABA(B) actions in neocortical neurons of the rat in vitro. Neuroscience 93:1241-1249.

Floeter MK, Rothwell JC (1999) Releasing the brakes before pressing the gas pedal. Neurology 53:664-665.

Hammond G, Vallence A-M (2007) Modulation of long-interval intracortical inhibition and the silent period by voluntary contraction. Brain Res 1158:63-70.

Hess A, Kunesch E, Classen J, Hoeppner J, Stefan K, Benecke R (1999) Task-dependent modulation of inhibitory actions within the primary motor cortex. Exp Brain Res 124:321-330.

Kouchtir-Devanne N, Capaday C, Cassim F, Derambure P, Devanne H (2012) Taskdependent changes of motor cortical network excitability during precision grip compared to isolated finger contraction. J Neurophysiol 107:1522-1529.

Kujirai T, Caramia MD, Rothwell JC, Day BL, Thompson PD, Ferbert A, Wroe S, Asselman $\mathrm{P}$, Marsden CD (1993) Corticocortical inhibition in human motor cortex. J Physiol 471:501-519.

Ortu E, Deriu F, Suppa A, Tolu E, Rothwell JC (2008) Effects of volitional contraction on intracortical inhibition and facilitation in the human motor cortex. J Physiol 586:5147-5159.

Reynolds C, Ashby P (1999) Inhibition in the human motor cortex is reduced just before a voluntary contraction. Neurology 53:730-735. 
Ridding MC, Rothwell JC (1999) Afferent input and cortical organisation: a study with magnetic stimulation. Exp Brain Res 126:536-544.

Ridding MC, Taylor JL, Rothwell JC (1995) The effect of voluntary contraction on corticocortical inhibition in human motor cortex. J Physiol 487 ( Pt 2):541-548.

Rosenkranz K, Rothwell JC (2003) Differential effect of muscle vibration on intracortical inhibitory circuits in humans. J Physiol 551:649-660.

Rosenkranz K, Rothwell JC (2004) The effect of sensory input and attention on the sensorimotor organization of the hand area of the human motor cortex. J Physiol 561:307-320.

Sailer A, Molnar GF, Cunic DI, Chen R (2002) Effects of peripheral sensory input on cortical inhibition in humans. J Physiol 544:617-629.

Sanger TD, Garg RR, Chen R (2001) Interactions between two different inhibitory systems in the human motor cortex. J Physiol 530:307-317.

Udupa K, Ni Z, Gunraj C, Chen R (2009) Interactions between short latency afferent inhibition and long interval intracortical inhibition. Exp Brain Res 199:177-183.

Vallence A-M, Schneider LA, Pitcher JB, Ridding MC (2014) Long-interval facilitation and inhibition are differentially affected by conditioning stimulus intensity over different time courses. Neurosci Lett 570:114-118.

Valls-Solé J, Pascual-Leone A, Wassermann EM, Hallett M (1992) Human motor evoked responses to paired transcranial magnetic stimuli. Electroencephalogr Clin Neurophysiol 85:355-364.

Wassermann EM, Samii A, Mercuri B, Ikoma K, Oddo D, Grill SE, Hallett M (1996) Responses to paired transcranial magnetic stimuli in resting, active, and recently activated muscles. Exp Brain Res 109:158-163. 




InterStimulus Interval (arbitrary units)

Figure 1. Theoretical curve describing the time-dependent modulation of the conditioned MEPs as a function of the time interval between the conditioning and test pulses. After the period of LICI, the conditioned MEP's return to baseline corresponds to LCD. The LCD may then be followed in some cases by a net increase in cortical excitability (corresponding to LICF). 


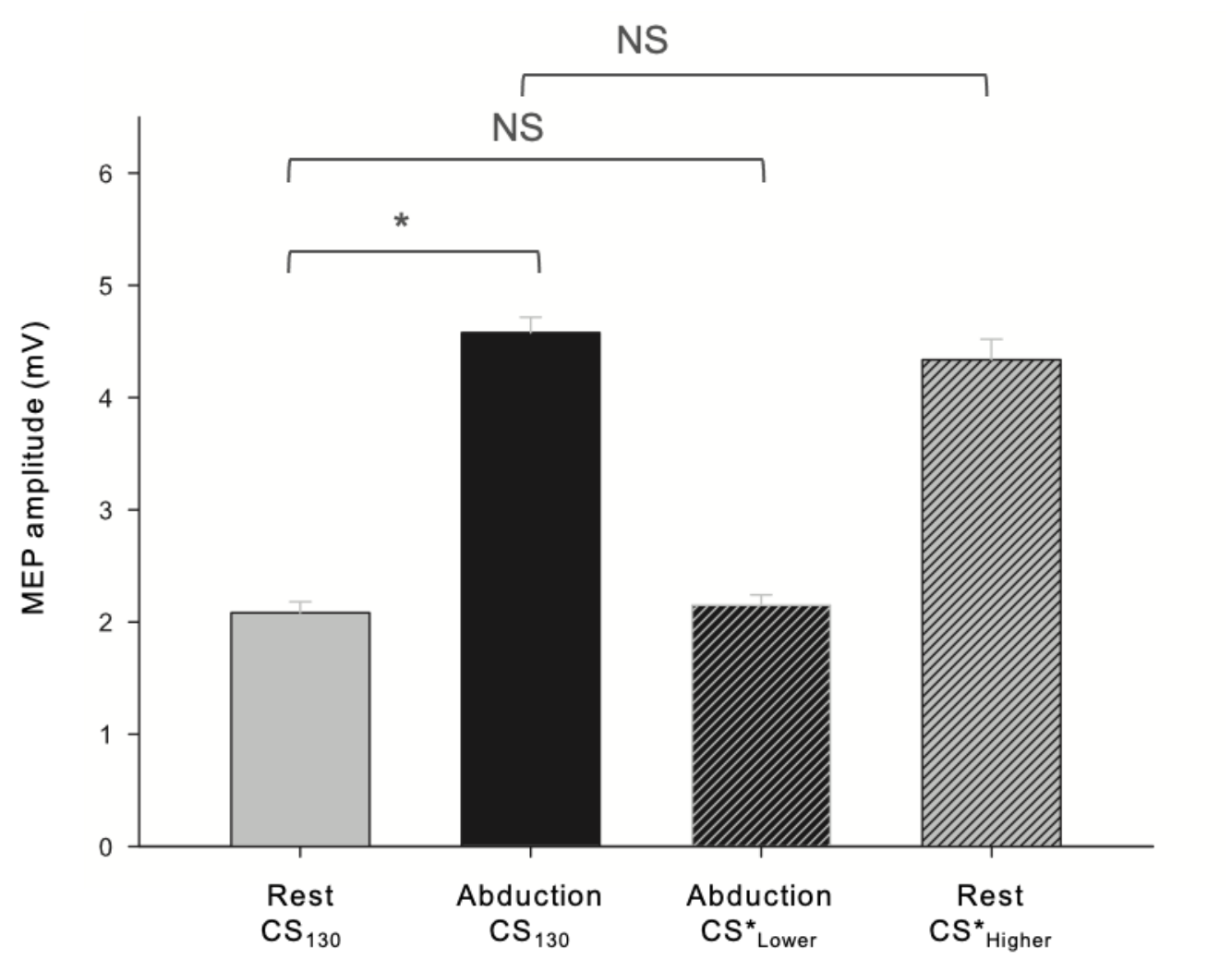

Figure 2. Mean amplitude of the motor response evoked by the conditioning stimulus in the various series. As expected, MEPs evoked at $\mathrm{CS}_{130}$ were significantly different between rest (first column) and abduction (second column). As can be observed, when the CS intensity was reduced in the abduction task (third column), the resulting MEP matched that induced by $\mathrm{CS}_{130}$ at rest. In a similar way, increasing the CS intensity in the rest condition (fourth column) resulted in an MEP whose amplitude matched that induced by $\mathrm{CS}_{130}$ in the abduction task. The error bars indicate standard errors and the asterisk indicates a significant difference at $\mathrm{p}<0.05$. 

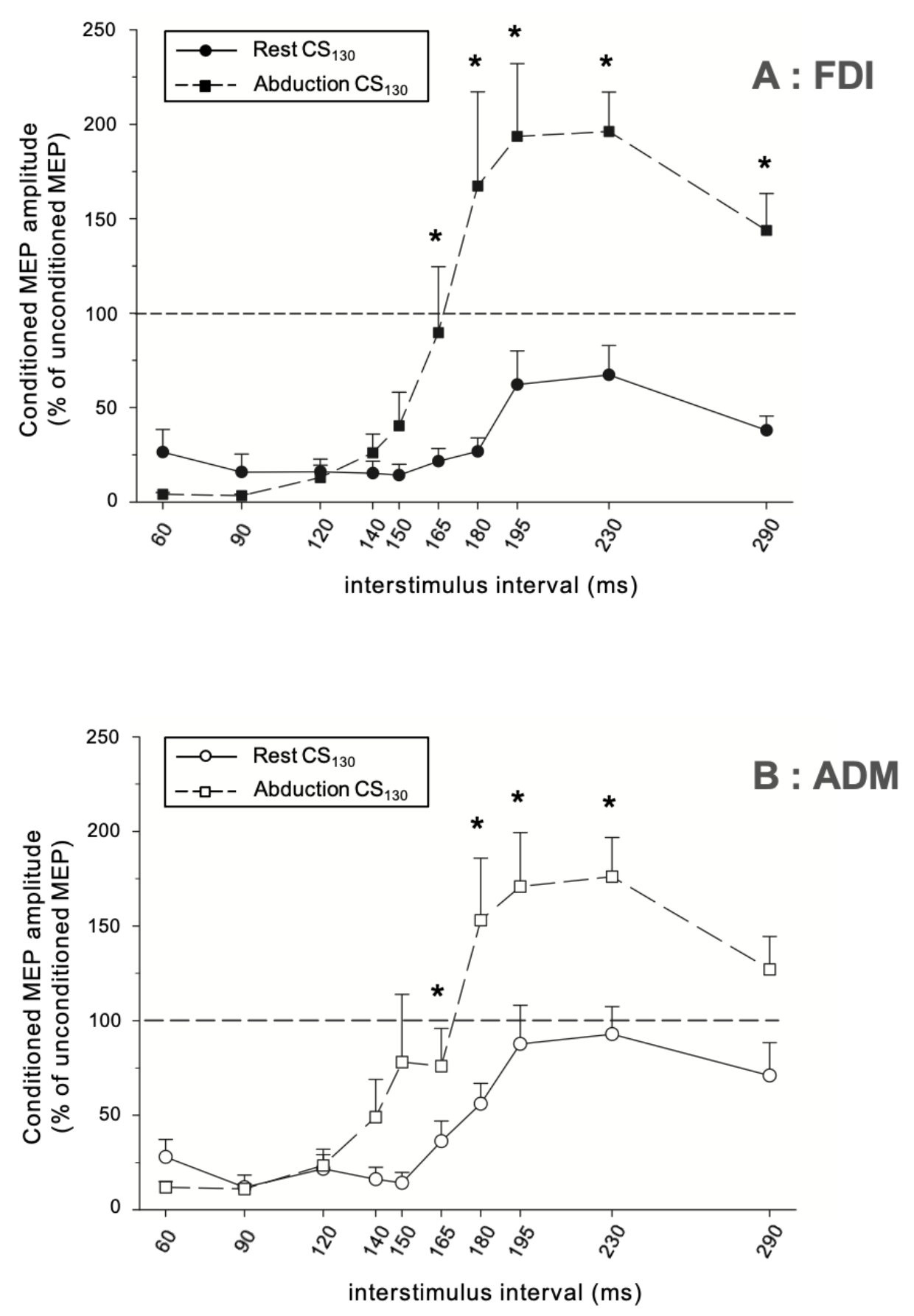

Figure 3. Change over time in the conditioned MEP amplitude at rest (circles, solid line) and during an abduction task (squares, dashed line) for the FDI (A) and the ADM (B) as a function of the ISI. Under both conditions, the CS intensity was set to $130 \%$ RMT. The horizontal dashed line represents the non-conditioned control MEP. Error bars indicate standard errors. Significant differences $(\mathrm{p}<0.05)$ between rest and abduction are indicated by an asterisk. 

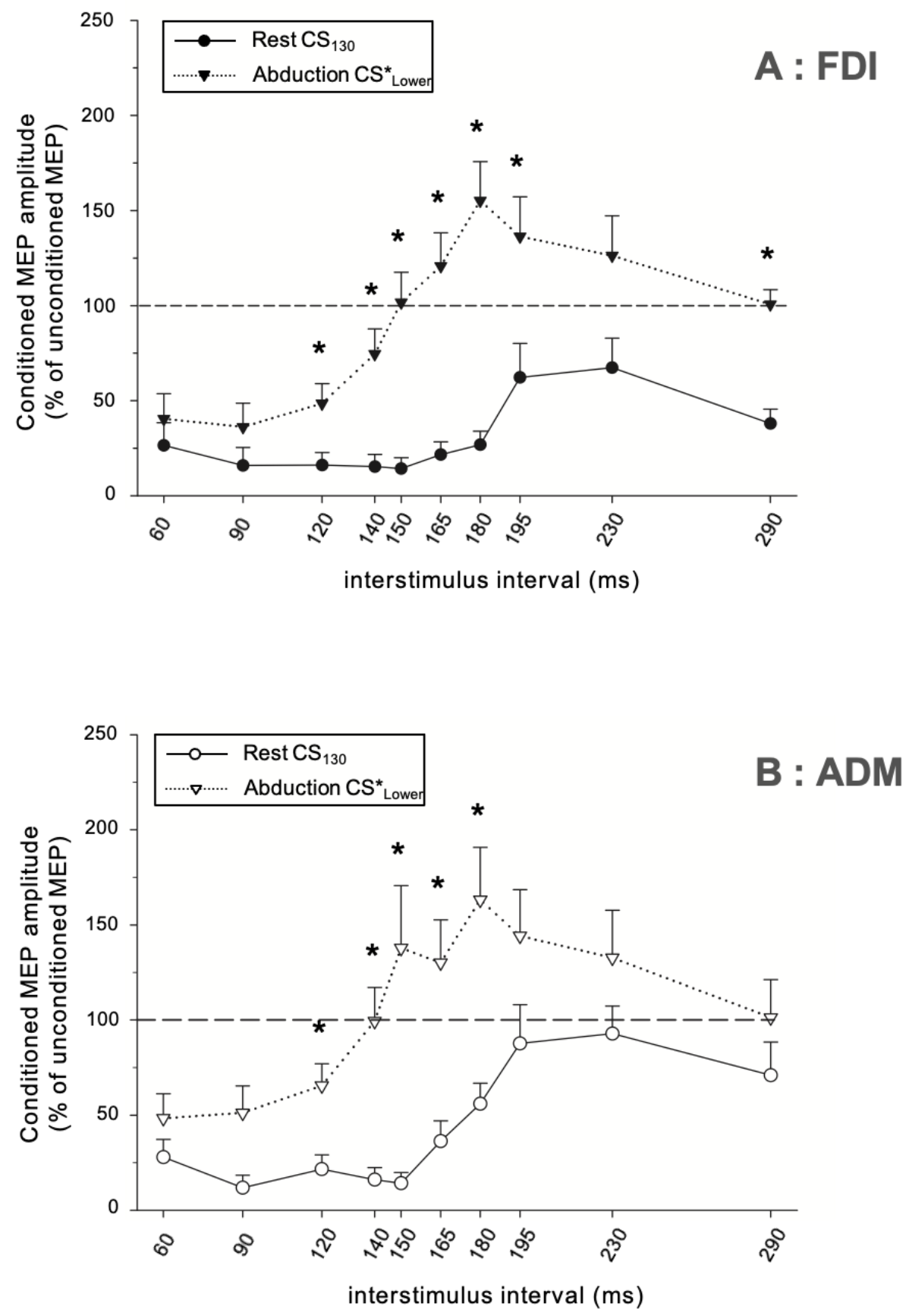

Figure 4. Change over time in the conditioned MEP amplitude at rest (circles, solid line) and during an abduction task (triangles, dotted line) in the FDI (A) and the ADM (B) as a function of the ISI. The CS intensity was set 130\% RMT at rest and then adjusted in abduction (CS* ${ }_{\text {Lower }}$ ) so that the induced MEP matched that obtained at rest. The horizontal dashed line represents the non-conditioned MEP. Error bars indicate standard errors. Significant differences $(\mathrm{p}<0.05)$ between rest and abduction are indicated by an asterisk. 

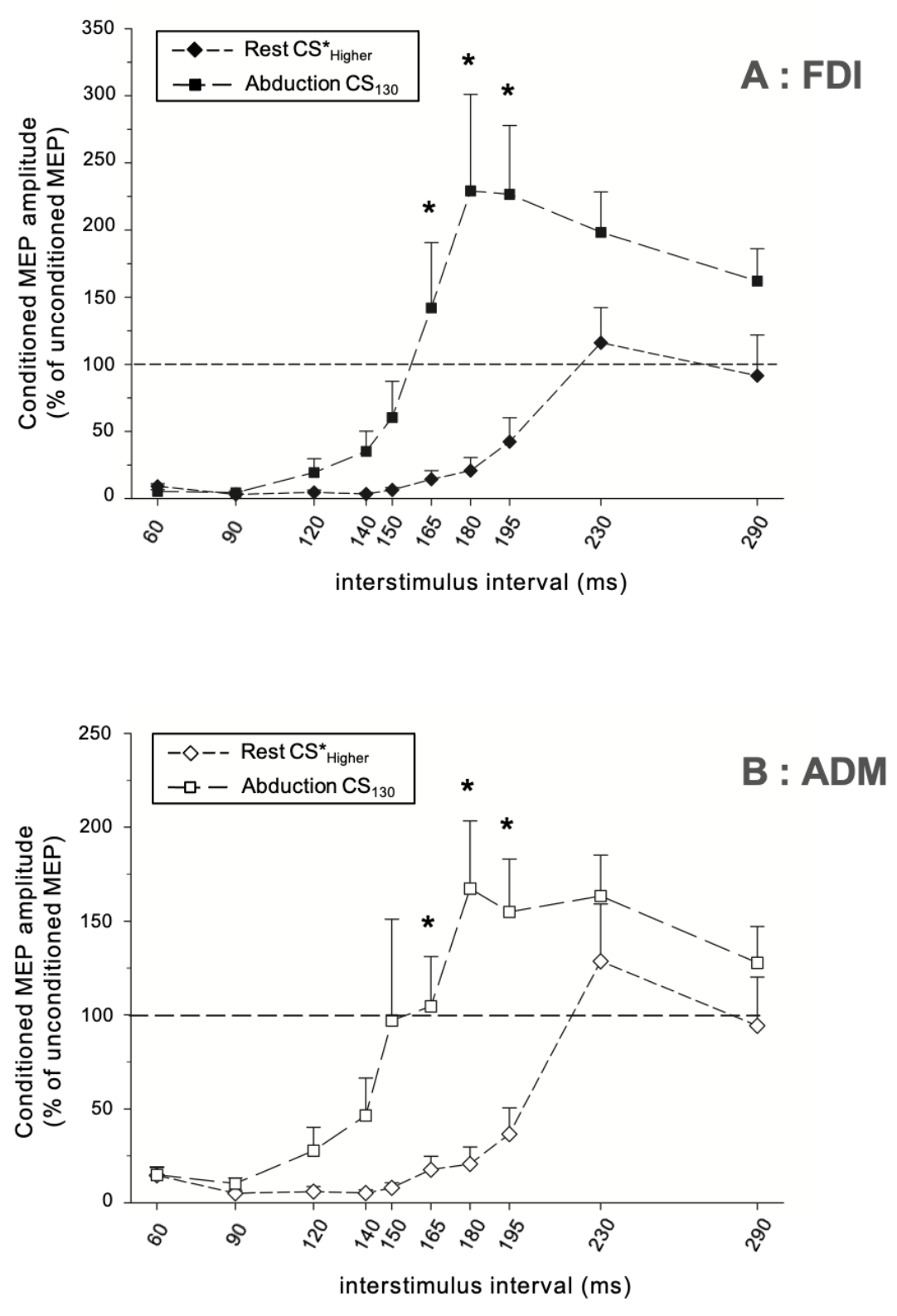

Figure 5. Change over time in the conditioned MEP amplitude at rest (diamonds, dashed line) and during an abduction task (squares, dashed line) in the FDI (A) and the ADM (B) as a function of the ISI. The CS intensity was set to 130\% RMT in the abduction task, and then adjusted at rest ( $\left.\mathrm{CS}^{*}{ }_{\text {Higher }}\right)$ so that so that the induced MEP matched that obtained during abduction. The horizontal dashed line represents the non-conditioned MEP. Note that the upper graph has larger vertical scale. Error bars indicate standard errors. Significant differences $(\mathrm{p}<0.05)$ between rest and abduction are indicated by an asterisk. 

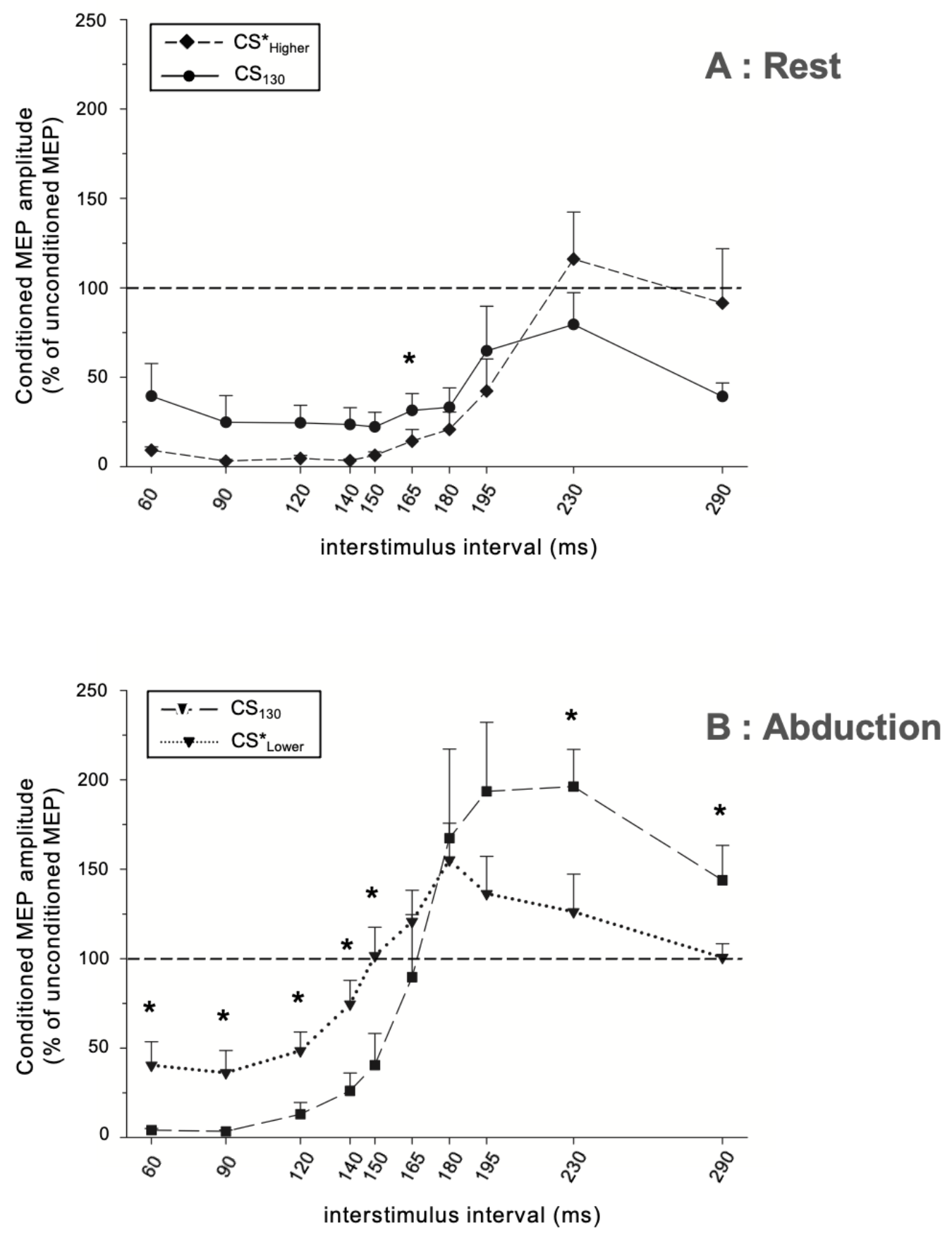

Figure 6. Influence of CS intensity on the overall time-dependent evolution of LICI , LCD and LICF in the FDI muscle at rest (upper graph) and during active abduction (lower graph). Error bars indicate standard errors. Significant differences $(\mathrm{p}<0.05)$ between the conditioned MEPs after $\mathrm{CS}_{130}$ and $\mathrm{CS}^{*}{ }_{\text {Higher }}$ (upper graph) or after $\mathrm{CS}_{130}$ and $\mathrm{CS}^{*}{ }_{\text {Lower }}$ (lower graph) are indicated by an asterisk. 
Tables

\begin{tabular}{|c|c|c|c|c|}
\hline ISI (ms) & $\begin{array}{c}\text { Rest } \\
\mathrm{CS}_{130}\end{array}$ & $\begin{array}{c}\text { Rest } \\
\text { CS }^{*}{ }_{\text {Higher }}\end{array}$ & $\begin{array}{c}\text { Abduction } \\
\text { CS }_{130}\end{array}$ & $\begin{array}{c}\text { Abduction } \\
\text { CS }_{\text {Lower }}\end{array}$ \\
\hline 60 & $*$ & $*$ & $*$ & $*$ \\
\hline 90 & $*$ & $*$ & $*$ & $*$ \\
\hline 120 & $*$ & $*$ & $*$ & NS \\
\hline 140 & $*$ & $*$ & $*$ & NS \\
\hline 150 & $*$ & $*$ & NS & NS \\
\hline 165 & $*$ & $*$ & NS & $*$ \\
\hline 180 & $*$ & $*$ & $*$ & $*$ \\
\hline 195 & NS & NS & $*$ & NS \\
\hline 230 & NS & NS & $*$ & NS \\
\hline 290 & $*$ & & & \\
\hline
\end{tabular}

Table 1 : Results of the one-sample t-test used to compare the conditioned MEP amplitude with the control MEP (100\%) for the FDI muscle $\left(^{*}: \mathrm{p}<0.05\right.$; NS : non significant) 


\begin{tabular}{|c|c|c|c|c|}
\hline ISI (ms) & $\begin{array}{c}\text { Rest } \\
\mathrm{CS}_{130}\end{array}$ & $\begin{array}{c}\text { Rest } \\
\mathrm{CS}^{*}{ }_{\text {Higher }}\end{array}$ & $\begin{array}{c}\text { Abduction } \\
\text { CS }_{130}\end{array}$ & $\begin{array}{c}\text { Abduction } \\
\text { CS }{ }_{\text {Lower }}\end{array}$ \\
\hline 60 & $*$ & $*$ & $*$ & $*$ \\
\hline 90 & $*$ & $*$ & $*$ & $*$ \\
\hline 120 & $*$ & $*$ & NS & NS \\
\hline 140 & $*$ & $*$ & NS & NS \\
\hline 150 & $*$ & $*$ & NS & NS \\
\hline 165 & $*$ & $*$ & $*$ & $*$ \\
\hline 180 & $*$ & $*$ & NS & NS \\
\hline 195 & NS & NS & NS & NS \\
\hline 230 & NS & NS & NS & NS \\
\hline 290 & NS & & & \\
\hline
\end{tabular}

Table 2 : Results of the one-sample t-test used to compare the conditioned MEP amplitude with the control MEP $(100 \%)$ for the ADM muscle $\left(^{*}: \mathrm{p}<0.05\right.$; NS : non significant) 